\title{
Solid phase epitaxy of molecular beam deposited amorphous GaAs on S:
}

\author{
Kiyohiko Yoshino, Kouichi Murakami, Shin Yokoyama, and Kohzoh Masuda \\ Institute of Materials Science, University of Tsukuba, Tsukuba Academic City, Ibaraki 305 , Japan
}

(Received 6 January 1989 ; accepted for publication 7 April 1989)

\begin{abstract}
Solid phase epitaxial ( $S P E$ ) crystallization of amorphous GaAs on (100) Si tilted by $4^{\circ}$ toward (011) formed by molecular beam deposition (MBD) was first achieved by cw $\mathrm{Kr}$ laser irraciation for short durations. The ratio of $\mathrm{As}$ to $\mathrm{Ga}(y / x)$ in deposited amorphous $\mathrm{Ga}_{x} \mathrm{As}_{y}$ films was varied from 0.4 to 1.2. Buring the laser iradiation, movement of the amorphous/ crystalline interface was measured using time-resolved optical reflectivity (TROR). It was found from TROR and micro-Raman scattering measurements that hetero-SPE is attained in samples with $\mathrm{As} / \mathrm{Ga}$ ratios ranging from 0.8 to 1.1 and that the interface roughness is larger than that observed in homo-SPE (e.g., MBD GaAs on GaAs and $\mathrm{P}^{+}$ion-implanted GaAs).
\end{abstract}

Solid phase epitarial (SPE) growth ${ }^{1}$ is a unique technology for growth of thin films at relatively low temperatures. Recently, many studies on the formation of GaAs films on Si have been performed using molecular beam epitaxy (MBE) ${ }^{2,3}$ or metalorganic chemical vapor deposition (MOCVD) ${ }^{4}$ methods. However, there are several problems associated with these growth techniques including (1) thermal stress caused by the diference of themal expansion between the film and the substrate, (2) degradation of surface morphology during deposition, and (3) generation of a large density of dislocations. In order to overcome these problems, SPE growth has been found to be useful and interesting because it is a low-temperature process. If a localized area of amorphous GaAs is selectively crystallized by using a cw laser beam, warping of the Si wafer can be avoided and a flat surface can be obtained.

In the two-step growth process, ${ }^{4}$ an amorphous GaAs buffer layer is first deposited at a low temperature $\left(<120^{\circ} \mathrm{C}\right.$ ). The layer has good surface morphology because the deposited atoms do not easily migrate on the Si surface. ${ }^{4.5}$ Before the device layer is deposited, the wafer is annealed to crystallize the buffer layer by $\mathbf{S P E}$. Good surface morphology is maintained even after annealing.

In this letter we report the hetero-SPE of molecular beam deposited (MBD) ${ }^{5}$ amorphous GaAs on Si by laser beam irradiation. Hetero-SPE has been investigated by using time-resolved optical reflectivity (TROR) ${ }^{6-9}$ measurements for SPE dynamics and by using micro-Raman scattering measurements for evaluation of crystallinity after SPE growth. The As to Ga ratios over which hetero-SPE growth is possible have been determined, and the roughness of the amorphous/crystalline interface during the SPE growth has been measured.

The substrate was a (100) Si wafer tilted by $4^{\circ}$ toward (011). Before loading the substrate into a MBE chamber, the Si wafer was oxidized and etched in solutions of boiling $\mathrm{HNO}_{3}$ and $50 \%$ HF. This process was repeated five times. Finally to protect the surface, the Si wafer was oxidized in a solution of boiling $\mathrm{HCl}: \mathrm{H}_{2} \mathrm{O}_{2}: \mathrm{H}_{2} \mathrm{O}=3: 1: 1$. In the $\mathrm{MBE}$ chamber at $10^{-9}$ Torr, the $\mathrm{SiO}_{2}$ layer was removed by thermal etching at $800^{\circ} \mathrm{C}$. The structural change in the Si surface was observed by reflection high-energy electron diffraction (RHEED). After themal etching, a $2 \times 1$ streak pattern of the (100) Si surface was observed. A monolayer of As atoms was deposited on the cleaned substrate at $700^{\circ} \mathrm{C}$ and $3.1 \times 2$ pattern $^{2}$ was observed from the deposited layer. Following the deposition of the As fim, the amorphous Ga $A s_{y}$ layer was deposited. The deposition was performed at $80^{\circ} \mathrm{C}$, and the thickness was $80 \mathrm{~mm}$. The ratio $(y / x)$ was determined using Auger electron spectroscopy.

Laser irradiation of the heterostructure was petformed in an air ambient. An encapsulation layer was not used because the duration of irradiation was very short (from $10 \mathrm{~ms}$ to $20 \mathrm{~s}$ ). A cw $\mathrm{Kr}$ laser $(\lambda=647.0 \mathrm{~nm}$ and $676.0 \mathrm{~nm}$ ) beam was used to heat the sample and a $\mathrm{cW}$ He-pie laser $(\lambda=632.8 \mathrm{~nm})$ probe beam was used for the TROR measurement. The $\mathrm{cw} \mathrm{Kr}$ laser beam was elliptically focused to a spot $\sim 60 \mu \mathrm{m}$ in the long axis and $\sim 40 \mu \mathrm{m}$ in the short axis, and the irradiation time was controlled by a mechanical shutter. The size of the probe laser beam was $5 \mu \mathrm{m}$. The heating laser power was varied from 3.5 to $6.5 \mathrm{~W}$ and various irradiation times were selected for each laser power. The irradiation time was selected to be between the time when the $\$$ PE growth is completed and the time when surface degradation begins to occur.

Raman spectra ${ }^{3}$ were measured in a backscattering geometry using a JASCO TRS-501S spectrometer with a $R$ MRS-11B microscope. The scattered light was detected by using photon counting techniques at $-35^{\circ} \mathrm{C}$, and the detected signal was processed with a multichannel analyzer. An Ar laser with a wavelength of $514.5 \mathrm{~nm}$ served as the excitation source for the Raman measurements. The penetration depth at $514.5 \mathrm{~nm}$ is approximately $100 \mathrm{~nm}$ in the crystalline GaAs. The Ar laser beam was focused down to about 1 km and the center in each crystallized region was irradiated by the focused beam.

In order to investigate the dependence of the SPE qual:ty on the As/Ga ratio $(y / x)$, amorphous $\mathrm{Ga}_{x} \mathrm{As}$, samples with different $\mathrm{As} / \mathrm{Ga}$ ratios ranging from 0.4 to 1.2 were irradiated with $\mathrm{Kr}$ laser. Typical TROR signals are shown in Fig. 1. The heating laser power was kept at $5.8 \mathrm{~W}$. The onset and end times of the laser irradiation are indicated by arrows in Fig. 1 . The temperature of the GaAs layer is immediately raised within $1 \mathrm{~ms}$ from the onset. ${ }^{6,7}$ The temperature during laser irradiation was estimated to be $380^{\circ} \mathrm{C}$ by simply comparing the SPE velocity measured at $y / x=1.0$ with the rate 


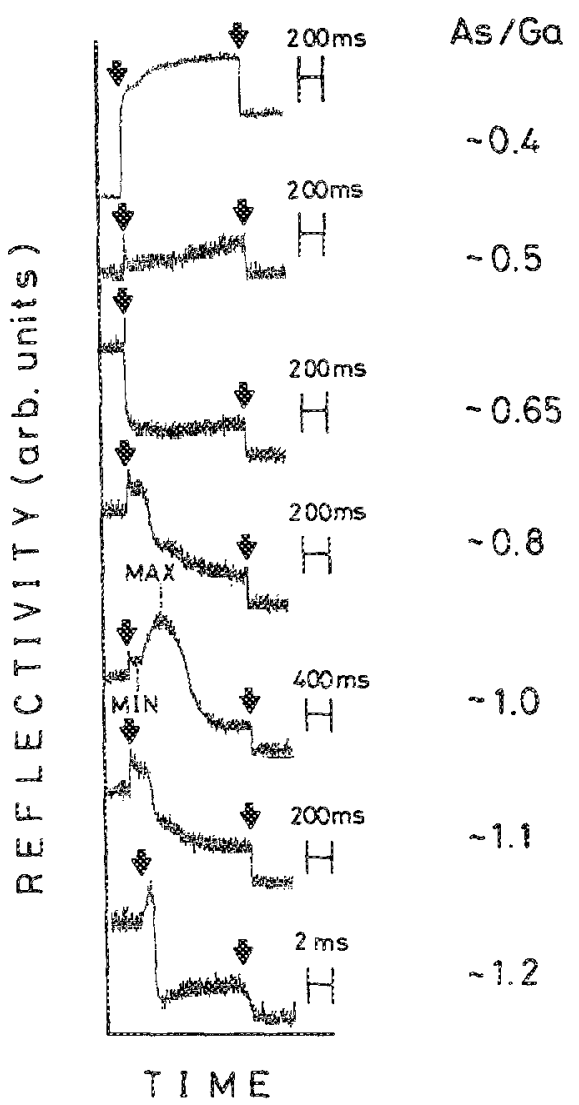

FIG. 1. TROR signais for various amorphous GaAs on Si samples with As/ Ga ratios from 0.4 to 1.2 during laser irradiation. Laser power is $5.8 \mathrm{~W}$ and the temperature is estimated to be $\sim 380^{\circ} \mathrm{C}$.

versus temperature cata obtained previously for amorphous GaAs on GaAs. ${ }^{5,6,8,9}$ The estimated temperature is higher than those ( $\leqslant 300^{\circ} \mathrm{C}$ ) previously used for SPE of amorphous GaAs on GaAs. ${ }^{5}$ A clear interference indicating SPE growth was observed only for the case of $y / x=1.0$, while small interferences were observed for both cases of 0.8 and 1.1. For other ratios such an interference was not evident, suggesting that another type of crystalization such as polycrystallization had occurred. It is found that SPE growth is successfully attained when the $\mathrm{As} / \mathrm{G}$ a ratio is between 0.8 and 1.1. This range is similar with that reported for SPE of amorphous GaAs on GaAs. ${ }^{5}$ It is also noted that for $y /$ $x=1.2$, the change in reflectivity is very fast $(<1 \mathrm{~ms})$ and can be observed even at lower laser powers (1.2 W).

At the ratio $y / x$ of 1.0 , laser irradiation with various laser powers was performed in order to change the annealing temperature. Figure 2 shows TROR signals for various laser powers ranging from 3.5 to $6.0 \mathrm{~W}$. As the laser power is increased, the amplitude of the TROR signal becomes smaller. It was observed in a cross-section view obtained by a transmission electron microscopy (TEM) measurement that formation of polycrystalline material ahead of the interface does not occur, but SPE with a rough interface takes place. These indicate that the roughness of an amorphous/ crystalline interface increases with increasing the temperature. However, the surface morphology was not changed at higher laser powers used here.

In Table 1 , the interface roughness derived from TROR signals is compared for three types of amorphous GaAs: (1)

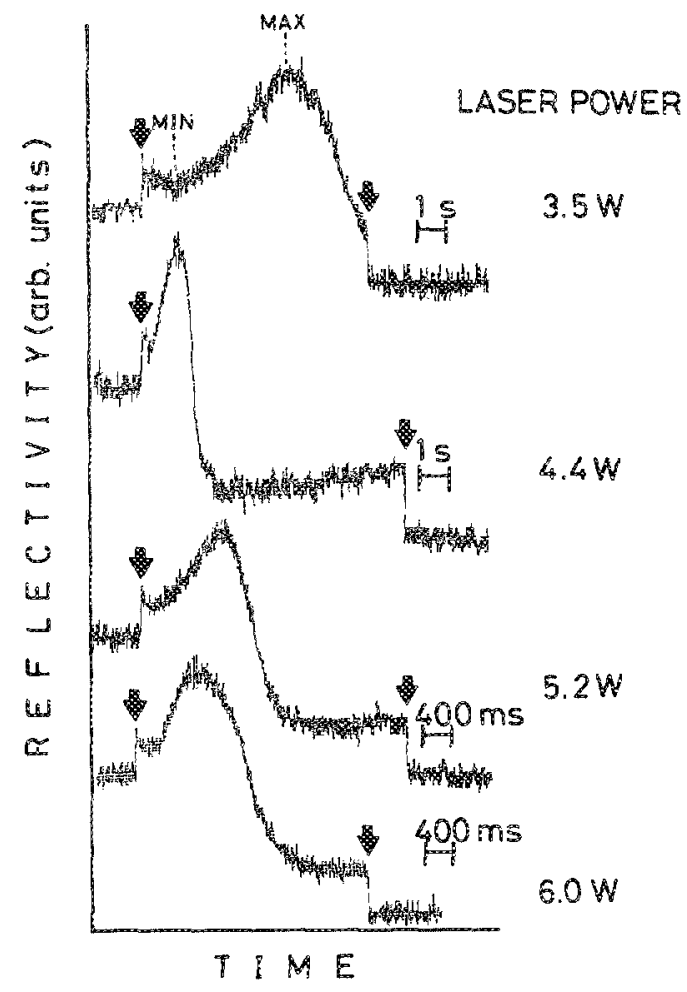

HG. 2. Easer power dependence of TROR signals for amorphous $G a_{1} A S_{1}$ on Si. Laser power is varied from 3.5 to $6.0 \mathrm{~W}$. The amplitude of TROR signal is strongly correlated with the roughness of amorphous/crystalline interface.

amorphous GaAs produced by $\mathrm{p}^{+-}$implantation ( $50 \mathrm{kV}$, $2 \times 10^{15} \mathrm{~cm}^{2}$ ), (2) MBD amorphous GaAs on crystalline GaAs, and (3) MBD amorphous GaAs on \$i. The thicknesses of the amorphous layers are $\sim 80, \sim 65$, and $\sim 80 \mathrm{~nm}$, respectively. The parameter in Table $I$ is an estimated annealing temperature. We evaiuated the interface roughness by a simple model in which the interface roughness is represented by Gaussian distribution 6,9 with standard deviation being cefined as the interface roughness. The interface roughness of MBD amorphous GaAs on $\mathrm{Si}$ is found to be about two times larger than the others at the same temperature. This enhancement of roughness in the $\mathrm{GaAs} / \mathrm{Si}$ system seems to be due to the initial heterointerface inciuding the diference of lattice constant. In studies of the initial growth of MBE GaAs on tilted Si, it is known from high-resolution TEM that misfit dislocations are generated at the step edge which exist every $\sim 4 \mathrm{~nm} .{ }^{10}$ Furthermore, there is $4 \%$ lattice

TABLE I. Interface roughness $\mathscr{S}(\mathrm{nm})$ for three types of amorphous GaAs. In the case of MBD GaAs on Si, the roughness is much larger than other homo-SpE cases at the same temperature.

\begin{tabular}{cccc}
\hline $\begin{array}{c}T_{\text {sub }} \\
(\mathrm{C})\end{array}$ & $\begin{array}{c}P^{+} \text {impianted } \\
\text { GaAs }\end{array}$ & MBD GaAs/GaAs & MBD GaAs/Si \\
\hline 340 & 35 & $<35$ & 40 \\
350 & 45 & 35 & 70 \\
370 & 45 & $<45$ & 100 \\
380 & 50 & 40 & 110 \\
400 & 60 & 50 & $\ldots$ \\
450 & 90 & $>50$ & $\ldots$ \\
\hline
\end{tabular}




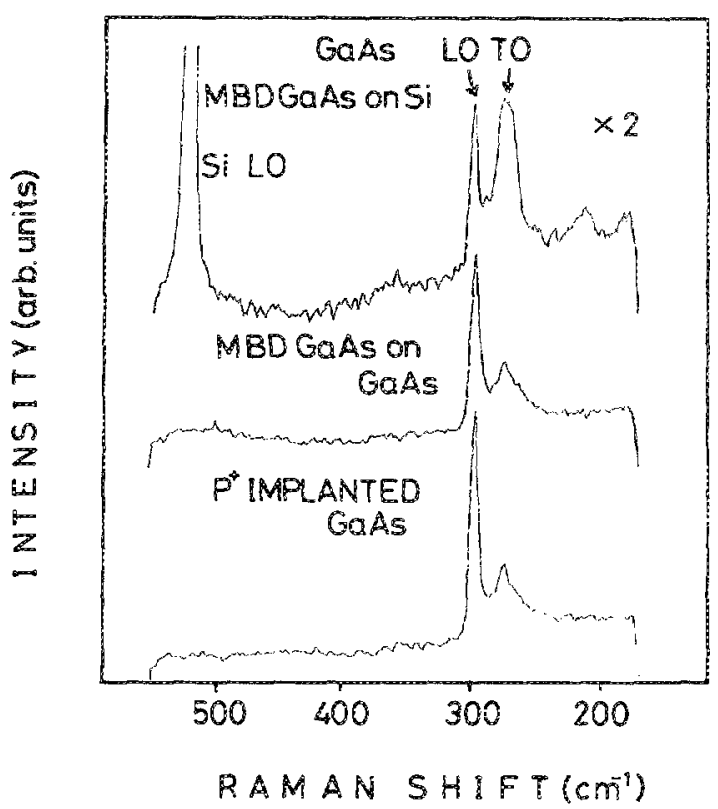

FIG. 3. Typical Raman spectra for MBD and ion-implanted GaAs crystallized by SPE. Amorphous GaAs on Si was annealed with a $6.5 \mathrm{~W} \mathrm{Kr}$ laser beam for $20 \mathrm{~s}$. Amorphous GaAs on GaAs substrate and ion-implanted amorphous GaAs layer were annealed with $1.8 \mathrm{~W}$ for $1.0 \mathrm{~s}$ and $2.2 \mathrm{~W}$ for $0.25 \mathrm{~s}$, respectively.

mismatch between Si and GaAs, so that the generation of dislocations every $\sim 10 \mathrm{~nm}^{i \prime}$ is needed to relax the lattice misfit.

We evaluated the crystallinity at the laser-annealed spot of amorphous GaAs by a micro-Raman scattering measurement. Typical Raman spectra are shown in Fig. 3. A. LO phonon line of the Si substrate is also observed. For this backscattering geometty with (100) GaAs, only LO phonons are allowed from the selection rules. However, the forbidden TO phonons are observed as well as LO phonons. The $T O$ phonons originate from regions with defects (e.g., twins and stacking faults) in the crystallized layer. With increasing laser power, the $\mathrm{LO}$-phonon signal becones Iarg$e r$, indicating better crystallinity. The best crystallinity obtained in this study (the top of Fig. 3 indicating the result for a sample annealed with $6.5 \mathrm{~W}$ for $20 \mathrm{~s}$ ) was still worse than that of the MBE GaAs layer on Si, " since the maximum laser output was limited to $6.5 \mathrm{~W}$. It was recently found that laser irradiation at high powers for shorter times ( $\leqslant 10 \mathrm{~ms}$ ) significantly improved the original crystallinity for the amorphous GaAs layer produced by $\mathrm{P}^{+}$implantation. ${ }^{1}$ This suggest that very rapid SPE will yield better crystallinity also for MBD amorphous GaAs on a Si system, although the interface roughness during SPE becomes larger.

In summary, we have investigated laser solid phase epitaxy of the MBD amorphous GaAs on Si using TROR and micro-Raman scattering measurements. From the TROR measurements on amorphous GaAs on Si samples with different ratios of $A$ s to $G a$, it is concluded that $S P E$ growth can be successfully achieved for ratios between 0.8 and 1.1 , while another type of crystalization occurred at other ratios. The roughness of an amorphous/crystalline interface for heteroSPE is larger than that for homo-SPE.

We would like to thank Professor M. Kawabe for preparation of MBD samples and Dr. R. Yoshizaki of the cryogenics center of University of Tsukuba for assistance with the micro-Raman scattering spectroscopy apparatus.

'L. Csepregi and J. W. Mayer, Phys. Lett. A \$4, 154 (1975).

${ }^{2}$ M. Kawabe and T. Ueda, Jpn. J. Appl. Phys. 25, L285 (1986).

${ }^{3}$ W. I. Wang, Appl. Phys. Lett. 44,1149 (1984).

${ }^{4}$ M. Akiyama, Y. Kâwarada, and K. Kaminishi, J. Cryst. Growth 68, 21 (1984)

S. Yokoyama, D. Yui, H. Tanigawa, H. Takasugi, and M. Kawabe, 3. Appl. Phys. 62, 1808 (1987).

"K. Takahasi, K. Murakami, K. Takita, ind K. Masuda, Proceedings of the 18th Symposium on Ion Implantation and Submicron Fabrication (Rikagaku Kenkyujo, Wako, 1987), p. 33.

'K. Murakami, Y. Tomiya, K. Takita, and K. Masuda, Appl. Phys. Lett. 45, 659 (1984).

${ }^{8}$ G. Olson, J. A. Roth, L. D. Hess, and J. Narayan, Proceedings of the MRS Symposium on Energy Beam Solid Interaction and Transient Thermal Processing (Materials Research Socicty, Pittsburgh, PA, 1984), p. 375.

${ }^{9} \mathrm{C}$. Licoppe, Y. I. Nissim, and C. Meriadec, J. Appl. Phys, 58, 3094 (1985).

${ }^{10}$ K. Takasugi, M. Kawabe, and Y. Bando, Jpn. J. Appl. Phys. 26, L584 (1987).

"K. Yoshino, K. Murakami, and K. Masuda, Jpn. J. Appl. Phys. 28, L368 (1989). 